\title{
Clinical characteristics and analysis of familial oral lichen planus in eight Chinese families
}

\author{
SHU-LAI LU ${ }^{1-3}$, XIANG-MIN QI ${ }^{1,3}$, GANG DONG $^{2}$, SHU-LAN CHEN ${ }^{2}$, \\ DA-WEI GUO ${ }^{2}$, YUN-LONG WANG ${ }^{4}$ and PI-SHAN YANG ${ }^{1,3}$ \\ ${ }^{1}$ Department of Periodontology, School of Stomatology, Shandong University, Jinan, Shandong 250012; \\ ${ }^{2}$ Oral Medical Center of Qingdao Municipal Hospital, Qingdao, Shandong 266071; \\ ${ }^{3}$ Shandong Provincial Key Laboratory of Oral Biomedicine, Shandong University, Jinan, Shandong 250012; \\ ${ }^{4}$ Gout Laboratory, The Affiliated Hospital of Qingdao University, Qingdao, Shandong 266003, P.R. China
}

Received June 16, 2015; Accepted July 28, 2016

DOI: 10.3892/etm.2016.3597

\begin{abstract}
Oral lichen planus (OLP) is one of the most common oral mucosa diseases; however, familial OLP is uncommon. The present study reported and analyzed patients with familial OLP $(n=18)$ in eight different Chinese families between January 1, 2012 and December 31, 2013. Parameters analyzed include gender, age at diagnosis, lesion distribution and lesion type. The follow-up period for each patient was a minimum of 1 year. In this survey, 18/88 individuals in the eight families were affected. Females were more frequently affected, and the female to male ratio for familial OLP (2.2:1) was higher compared with that previously reported for nonfamilial OLP (1.4:1). The age at diagnosis, lesion distribution and lesion type showed consistency with reports concerning nonfamilial OLP, with the exception of family VI, in which $4 / 5$ children had OLP/LP lesions and were of an early age at diagnosis. There were two families in which three generations had been affected by OLP. In addition, it appeared that patients of the same generation in the same family were of a similar age at diagnosis. No malignant or premalignant lesion was identified in the 18 individuals diagnosed with OLP from the eight families. The present study supports the hypothesis that genetic predisposition may serve a role in the etiology of OLP.
\end{abstract}

Correspondence to: Dr Yun-Long Wang, Gout Laboratory, The Affiliated Hospital of Qingdao University, 16 Jiangsu Road, Qingdao, Shandong 266003, P.R. China

E-mail:wylapollo@163.com

Professor Pi-Shan Yang, Department of Periodontology, School of Stomatology, Shandong University, 44-1 West Wen Hua Road, Jinan, Shandong 250012, P.R. China

E-mail: yangps@sdu.edu.cn.

Key words: oral lichen planus, familial, etiology, genetic, premalignant

\section{Introduction}

Oral lichen planus (OLP) is a chronic inflammatory disease that affects oral mucosa of squamous cell origin (1), and is most commonly observed in middle aged women (2). The etiopathogenesis of OLP has yet to be elucidated. However, it is known to be associated with multiple factors and their immunological, endocrine, infective and psychological etiologies have been suggested in previous studies $(3,4)$. In recent years, the involvement of genetic factors in OLP has attracted increased attention.

OLP may occur in the buccal mucosa, tongue, gingiva, lip and palate, it may present in papular, reticular, plaque-like, atrophic, bullous and erosive forms (3-5), and frequently appears as a mixture of the forms (6). The lesions are typically multiple, symmetrical, bilateral and painless. However, erosive and atrophic lesions may cause pain or a burning sensation, and are considered to be premalignant (5).

OLP is one of the most common diseases of the oral mucosa, with a prevalence of 1-2\% (3,5,7-9). Typically, it presents sporadically, so familial OLP is rare. The current study reports cases of familial OLP in eight different Chinese families and analyzes their clinical characteristics.

The present study supports the notion that genetic predisposition may be important in OLP, and it also reminds us that OLP patient's relatives should be advised to perform check-ups in time in order to exclude or confirm OLP. Furthermore, the present study also provides samples for large-scale genetic studies of OLP in the future.

\section{Subjects and methods}

Ethics statement. The present study was approved by the Institutional Ethics Committee of Qingdao Municipal Hospital (Qingdao, China). Informed consent was obtained from one of the patients.

Subjects and inclusion criteria. A retrospective review was performed on eight families of individuals affected by familial OLP who were diagnosed between January 1, 2012 and December 31, 2013 at the Oral Medical Center of Qingdao Municipal Hospital. A diagnosis of familial OLP 
was concluded if the patient met the following criteria: i) The diagnosis of OLP was supported by clinical and pathological findings; and ii) at least two members of the patient's family had OLP.

Diagnosis criteria. The criteria for the diagnosis of OLP has been established by World Health Organization (10). Clinically, OLP frequently presents as multiple lesions with white striations that typically occur in the buccal mucosa, tongue and gingiva. The lesions may become erosive or atrophic, or may present in other clinical patterns (8). Histologically, OLP is characterized by a dense band-like lymphocytic infiltrate in the immediate sub-epithelial region with basal epithelial cell destruction (6). In the present study, the clinical and histological diagnosis was made by the same oral medicine doctor and the same pathology doctor, respectively.

Histological examination. In total, $\sim 0.5 \mathrm{~mm} \times 0.5 \mathrm{~mm}$ of mucosa with OLP lesion was incised under local anesthesia. The sample was fixed in $10 \%$ formalin for $24 \mathrm{~h}$, paraffin-embedded and sectioned into $4-\mu \mathrm{m}$ slices. Furthermore, it was dewaxed by gradient alcohol, rinsed with $\mathrm{H}_{2} \mathrm{O}$, dyed by hematoxylin and eosin, dehydrated by gradient alcohol, hyalinized by xylene, sealed by neutral gum and observed using a BX51 microscope (Olympus Corporation, Tokyo Japan).

Each individual that met the diagnosis criteria of familial OLP was surveyed and recorded. For each patient, the gender, age at diagnosis, lesion distribution and lesion type were recorded and analyzed, and the follow-up was a minimum of 1 year.

\section{Results}

Genetic predisposition. The pedigrees of the eight families are shown in Fig. 1. Six families had two affected members and two families had three affected members among the three generations. Fig 1 supports the hypothesis that OLP has a genetic predisposition.

Among the 88 family members, 18 individuals were affected (female, $n=13$; male, $n=5$ ), and the female to male ratio was 2.6:1, which was higher compared with nonfamilial OLP (1.4:1) (6).

Clinical characteristics. The clinical characteristics of the individuals with OLP from the eight families are presented in Table I. Patients' age at diagnosis ranged between 25 and 70 years old, and the majority of the patients were aged between 40 and 49 years.

With respect to the locations of the lesions, the buccal mucosa was the most frequently affected site, followed by the tongue, lip, gingiva and palate, and a number of patients had lesions at more than one location.

With respect to the lesion types, those with a reticular pattern accounted for the predominant clinical form of OLP, followed by erosive lesions, atrophic lesions, plaque-like lesions and papular lesions (Fig. 2). The majority of patients had multiple lesions, and bullous lesions were not detected (Table I).

No malignant/premalignant lesions were identified, and none of the patients had cutaneous lesions of LP. Other members of the families were clinically examined, however none showed mucosal or skin LP lesions, except family VI. This family included 5 children; two of the children had OLP, one had LP cutaneous lesions on the face and one had LP cutaneous lesions on the legs.

\section{Discussion}

Familial LP is uncommon, and its prevalence in a large sample study has been reported to be $1.5 \%$ (9), whilst familial OLP is even less common.

Compared with nonfamilial LP, familial LP is reported to be characterized by its early age of onset, its ability to become severe and chronic and to have atypical and widespread clinical presentation (11). In the present study concerning familial OLP, the clinical and pathological manifestations (Fig. 3) and parameters (gender, age at diagnosis, lesion distribution and lesion type) showed no difference compared with nonfamilial OLP, except in family VI, in which 4/5 children had OLP/LP lesions and were of an early age at diagnosis. In addition, it was identified that patients of the same generation in the same family were of a similar age at diagnosis.

In the eight families analyzed, once one member of the family had been initially diagnosed with OLP, other members were surveyed and further cases were identified. As papular, reticular and plaque-like lesions are typically asymptomatic, family members of individuals with OLP frequently fail to notice the existence of lesions prior to their clinical confirmation (6). Thus, the exact time of onset and duration is unknown to the patient. Consequentially, there is no record and analysis of the time of onset and duration in this study.

The etiology of OLP is associated with numerous factors. Previous studies have suggested that OLP is a T cell-mediated autoimmune disease $(3,12)$. Particular tendencies, such as smoking, alcohol consumption, drugs, eating spicy foods and bad hygiene, may exasperate symptoms of OLP $(3,4,6)$. In addition, psychological disorders, such as anxiety, depression and stress, are associated with OLP $(3,6,13)$. In addition, accidental abrasion of the oral mucosa by brushing of teeth causing an ulcer, pointed cusps, cracked teeth or worn dental restorations may worsen or trigger new lesions (4). Previously, OLP has been reported to be associated with systemic medical conditions, such as diabetes, hepatitis $\mathrm{C}$ viral infections, hypertension, ulcerative colitis, myasthenia gravis and lupus erythematosus $(2,3,4,11)$. Recently, several studies have revealed the genetic susceptibility of OLP through case control association testing (14-17).

The role of genetic factors in OLP have yet to be elucidated. In certain studies, the role of genetic predisposition was considered. Watanabe et al (18) concluded that human leukocyte antigen (HLA) served a role in the pathogenesis of OLP. Hedberg and Hunter (19) reported that epithelium affected by OLP was consistently positive for HLA-antigen D related. There are a number of clinical reports describing familial OLP and genetic predisposition. Wang et al (5) assessed a Chinese family affected with OLP and identified genetics as the cause for the disease. In addition, a report of OLP in three successive generations was highly indicative of a genetic predisposition to the disease (9). The aforementioned data, in addition to the current study, suggests that genetic predisposition serves a role in OLP.

The risk of malignant potential should be considered in OLP (1), which was classified as a premalignant condition by the World Health Organization in $1997(8,12)$. Currently, however, whether OLP is a premalignant condition remains 
Table I. Clinical features of 18 individuals from eight different families (I-VIII) with familial oral lichen planus.

\begin{tabular}{|c|c|c|c|c|c|c|c|c|c|}
\hline \multicolumn{2}{|c|}{ Number } & \multirow[b]{2}{*}{ Gender } & \multirow[b]{2}{*}{ Age at diagnosis } & \multicolumn{5}{|c|}{ Lesion distribution } & \multirow[b]{2}{*}{ Lesion type } \\
\hline Patient & Family & & & B & G & $\mathrm{T}$ & $\mathrm{L}$ & $\mathrm{P}$ & \\
\hline 1 & I & M & 60 & + & & + & & & R, E, PL, A \\
\hline 2 & I & $\mathrm{F}$ & 58 & + & & & & & $\mathrm{R}$ \\
\hline 3 & II & F & 49 & + & & + & & & R, PL \\
\hline 4 & II & $\mathrm{F}$ & 47 & + & & & & & PA \\
\hline 5 & III & M & 48 & + & & & & & $\mathrm{R}, \mathrm{E}, \mathrm{A}$ \\
\hline 6 & III & $\mathrm{F}$ & 25 & + & & & & & $\mathrm{R}$ \\
\hline 7 & IV & $\mathrm{F}$ & 70 & + & & + & & & $\mathrm{R}, \mathrm{E}, \mathrm{A}$ \\
\hline 8 & IV & $\mathrm{F}$ & 62 & + & & & & & $\mathrm{R}, \mathrm{E}$ \\
\hline 9 & IV & $\mathrm{F}$ & 38 & + & + & & & + & $\mathrm{R}, \mathrm{E}$ \\
\hline 10 & $\mathrm{~V}$ & M & 48 & + & + & + & & + & $\mathrm{R}, \mathrm{E}$ \\
\hline 11 & V & M & 46 & + & & & & & $\mathrm{R}$ \\
\hline 12 & VI & $\mathrm{F}$ & 31 & + & & & & & $\mathrm{R}$ \\
\hline 13 & VI & F & 30 & & & + & + & & PL, R \\
\hline 14 & VII & $\mathrm{F}$ & 58 & & & & + & & $\mathrm{R}, \mathrm{E}$ \\
\hline 15 & VII & $\mathrm{F}$ & 53 & + & & & & & $\mathrm{R}$ \\
\hline 16 & VIII & $\mathrm{F}$ & 62 & + & + & + & & & $\mathrm{R}, \mathrm{E}, \mathrm{A}$ \\
\hline 17 & VIII & $\mathrm{F}$ & 44 & + & & & & & $\mathrm{R}, \mathrm{E}$ \\
\hline 18 & VIII & M & 27 & + & & & + & & $\mathrm{R}$ \\
\hline
\end{tabular}

A, atrophic; B, buccal; E, erosive; F, female; G, gingiva; L, lip; M, male; P, palate; PA, papular; PL, plaque-like; R, reticular; T, tongue.

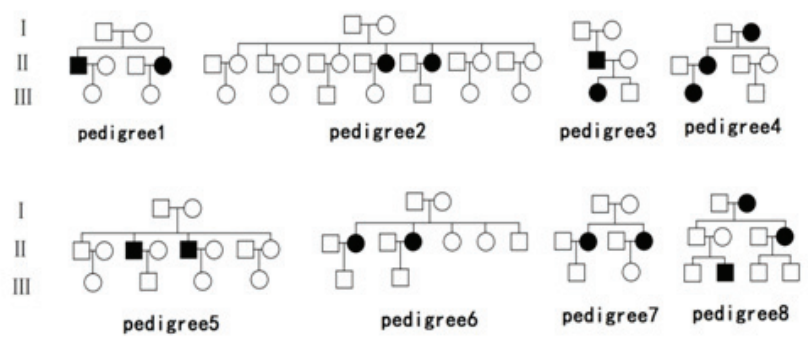

Figure 1. Pedigrees of the eight families with familial OLP.

controversial. Using stringent diagnostic criteria, a number of authors have found that patients with OLP are not at increased risk of oral squamous cell carcinoma. Accurso et al (20) evaluated loss of heterozygosity using laser capture microdissection or microsatellite instability and found that OLP exhibited a genetic profile that had greater similarity to a benign or reactive process compared with a premalignant/malignant one. Certain studies have concluded that several of the reported cases of oral carcinomas arising from OLP may have developed from lichenoid lesions with dysplasia $(8,18,21-24)$. However, there is a consensus supporting the potential for the malignant transformation of OLP, with a transformation rate from prospective studies ranging between 0.4 and $6.5 \%$. This is particularly true in erosive-type OLP, and in OLP occurring in the lateral and ventral side of the tongue $(12,25)$.

Whether familial OLP is a premalignant condition remains controversial. Wang et al (5) reported the case of a Chinese family with OLP affected by a severe form of oral reticular

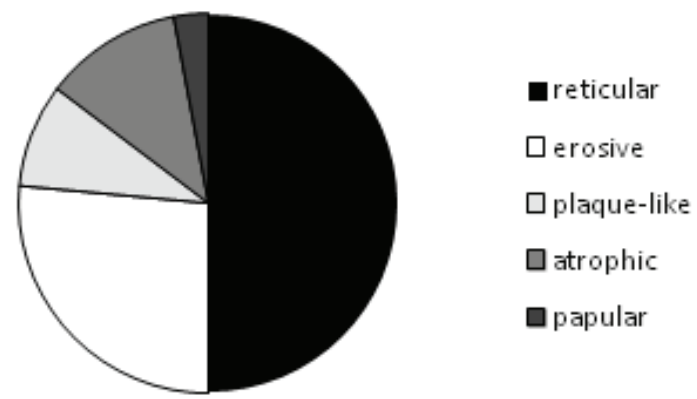

Figure 2. Different lesion types observed in eight Chinese families with familial oral lichen planus.

and erosive lesions, and 2 of the 5 affected individuals had developed oral cancer at an early age. However, Bermejo-Fenoll and López-Jornet (11) reported familial OLP in six families in Spain, none of whom developed oral cancer. The present report of familial OLP in eight Chinese families was in agreement with the aforementioned study. However, further investigation with a larger sample size for patients with familial OLP, and with longer-term clinical follow-up, is required.

The exact etiology of familial OLP has yet to be elucidated. The current study supports the notion that genetic predisposition may serve a role in the disease, and we speculate that this may be associated with the same family having similar habits, disposition and pedigree; however, the possibility of coincidence can not be ignored. Further studies with a larger sample of the population is essential to investigate genetic factors to reveal the pathogenesis of this rare condition. Whether there is a risk of 
A

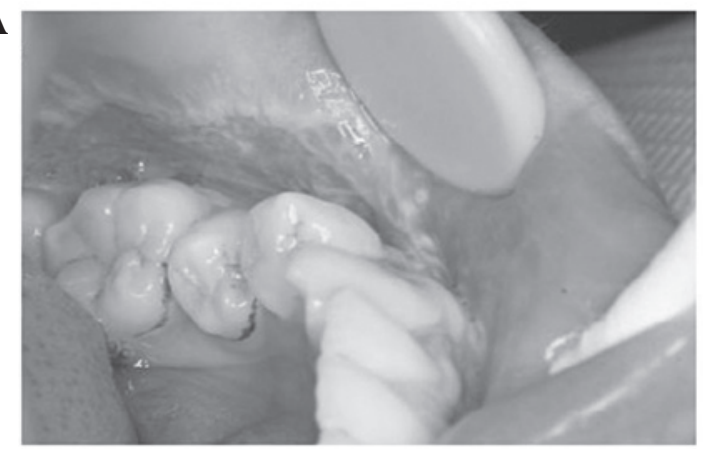

B

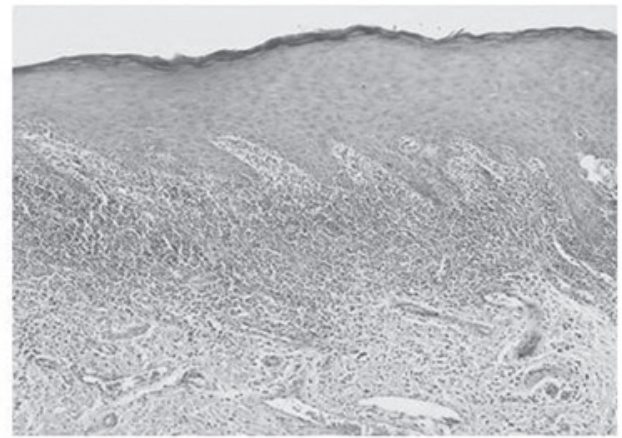

Figure 3. Clinical and histological images of oral lichen planus on the left buccal mucosa from patient 12 in family VI. (A) Clinical image: Lesions with white striations. (B) Histological image: A dense band-like lymphocytic infiltrate in the sub-epithelial region with basal layer hydropic generation (hematoxylin and eosin stain; magnification, $\mathrm{x} 100)$.

malignant transformation requires longer term follow-up and further research. Furthermore, when a patient is diagnosed with OLP, the possibility of canceration should be considered, and the patient's relatives should be advised to undergo a check-up in time to exclude or confirm presence of the disease, in order to achieve an early diagnosis and early treatment.

\section{Acknowledgements}

The present study was supported by funding from the National Natural Science Foundation of China (grant no. 81271141) and Qingdao Municipal Science and Technology Commission Major Project (grant no. 11-2-3-2-(7)-nsh).

\section{References}

1. Jin X, Wang J, Zhu L, Wang L, Dan H, Zeng X and Chen Q Association between -308 G/A polymorphism in TNF- $\alpha$ gene and lichen planus: A meta-analysis. J Dermatol Sci 68: 127-134, 2012.

2. Srinivas K, Aravinda K, Ratnakar P, Nigam N and Gupta S: Oral lichen planus-Review on etiopathogenesis. Natl J Maxillofac Surg 2: 15-16, 2011.

3. Barbosa NG, Silveira ÉJ, Lima EN, Oliveira PT, Soares MS and de Medeiros AM: Factors associated with clinical characteristics and symptoms in a case series of oral lichen planus. Int J Dermatol 54: e1-e6, 2015.

4. Di Stasio D, Guida A, Salerno C, Contaldo M, Esposito V, Laino L, Serpico R and Lucchese A: Oral lichen planus: A narrative review. Front Biosci (Elite Ed) 6: 370-376, 2014.

5. Wang Z, Yao H, Cui B, Ning G and Tang GY: Genetic linkage analysis of oral lichen planus in a Chinese family. Genet Mol Res 10: 1427-1433, 2011.

6. Sandhu SV, Sandhu JS, Bansal H and Dua V: Oral lichen planus and stress: An appraisal. Contemp Clin Dent 5: 352-356, 2014.

7. Ghapanchi J, Haghshenas MR, Ghaderi H, Amanpour S, Nemati V and Kamali F: Ctla-4 gene polymorphism in +49 a/g position: A case control study on patients with oral lichen planus. J Int Oral Health 6: 17-21, 2014.

8. Zhang L, Michelsen C, Cheng X, Zeng T, Priddy R and Rosin MP: Molecular analysis of oral lichen planus. A premalignant lesion? Am J Pathol 151: 323-327, 1997.

9. Singal A: Familial mucosal lichen planus in three successive generations. Int J Dermatol 44: 81-82, 2005.

10. Rad M, Hashemipoor MA, Mojtahedi A, Zarei MR, Chamani G, Kakoei S and Izadi N: Correlation between clinical and histopathologic diagnoses of oral lichen planus based on modified WHO diagnostic criteria. Oral Surg Oral Med Oral Pathol Oral Radiol Endod 107: 796-800, 2009.

11. Bermejo-Fenoll A and López-Jornet P: Familial oral lichen planus: Presentation of six families. Oral Surg Oral Med Oral Pathol Oral Radiol Endod 102: e12-e15, 2006.
12. Al-Nasser L and El-Metwally A: Oral lichen planus in Arab countries: A review. J Oral Pathol Med 43: 723-727, 2014.

13. Gavic L, Cigic L, Biocina Lukenda D, Gruden V and Gruden Pokupec JS: The role of anxiety, depression, and psychological stress on the clinical status of recurrent aphthous stomatitis and oral lichen planus. J Oral Pathol Med 43: 410-417, 2014.

14. Supic G, Kozomara R, Zeljic K, Stanimirovic D, Magic M, Surbatovic M, Jovic N and Magic Z: HMGB1 genetic polymorphisms in oral squamous cell carcinoma and oral lichen planus patients. Oral Dis 21: 536-543, 2015.

15. Jiang C, Yao H, Cui B, Zhou Y, Wang Y and Tang G: Association of interleukin 12A gene polymorphisms with oral lichen planus in Chinese population. J Oral Pathol Med 44: 602-606, 2015.

16. Wu D, Chen X, Dong C, Liu Q, Yang Y, He C, Wang J, Sun M and Wu Y: Association of single nucleotide polymorphisms in MPO and COX genes with oral lichen planus. Int J Immunogenet 42: 161-167, 2015.

17. Al-Mohaya MA, Al-Harthi F, Arfin M and Al-Asmari A: TNF- $\alpha$, TNF- $\beta$ and IL-10 gene polymorphism and association with oral lichen planus risk in Saudi patients. J Appl Oral Sci 23: 295-301, 2015.

18. Watanabe T, Ohishi M, Tanaka K and Sato H: Analysis of HLA antigens in Japanese with oral lichen planus. J Oral Pathol 15: 529-533, 1986.

19. Hedberg NM and Hunter N: The expression of HLA-DR on keratinocytes in oral lichen planus. J Oral Pathol 16: 31-35, 1987.

20. Accurso BT, Warner BM, Knobloch TJ, Weghorst CM, Shumway BS, Allen CM and Kalmar JR: Allelic imbalance in oral lichen planus and assessment of its classification as a premalignant condition. Oral Surg Oral Med Oral Pathol Oral Radiol Endod 112: 359-366, 2011.

21. Esquivel-PedrazaL,Fernández-CuevasL,Ruelas-VillavicencioAL, Guerrero-Ramos B, Hernández-Salazar A, Milke-García MP and Méndez-Flores S: Oral squamous cell carcinoma and lichen planus vs. lichenoid lesions. Case report. Rev Med Inst Mex Seguro Soc 54: 673-679, 2016 (In Spanish).

22. Gonzalez-Moles MA, Gil-Montoya JA, Ruiz-Avila I and Bravo M: Is oral cancer incidence among patients with oral lichen planus/ oral lichenoid lesions underestimated? J Oral Pathol Med: July 18, 2016 (Epub ahead of print) doi: 10.1111/jop.12480.

23. Mares S, Ben Slama L, Gruffaz F, Goudot P and Bertolus C: Potentially malignant character of oral lichen planus and lichenoid lesions. Rev Stomatol Chir Maxillofac Chir Orale 114: 293-298, 2013 (In French).

24. Fitzpatrick SG, Hirsch SA and Gordon SC: The malignant transformation of oral lichen planus and oral lichenoid lesions: A systematic review. J Am Dent Assoc 145: 45-56, 2014.

25. Lodi G, Scully C, Carrozzo M, Griffiths M, Sugerman PB and Thongprasom K: Current controversies in oral lichen planus: Report of an international consensus meeting. Part 2. Clinical management and malignant transformation. Oral Surg Oral Med Oral Pathol Oral Radiol Endod 100: 164-178, 2005. 